\title{
ON THE INVARIANTS OF A BINARY QUINTIC AND THE REALITY OF ITS ROOTS
}

\author{
By H. F. BAKER.
}

[Received October 19th, 1907.-Read November 14th, 1907.]

THE present note consists of three parts. In $\S 1$ it is shewn that the known rational relation connecting the four fundamental invariants of a binary quintic is resoluble; namely, that three rational invariants, each rational in the four fundamental invariants, can be taken, in terms of which each of the four fundamental invariants can be rationally expressed; thus all the rational invariants of the quintic are rationally expressible in terms of three, these being two absolute invariants and one of effective degree 2, the quotient of an integral invariant of degree 18 by one of degree 16 . In $\$ 2$ a discrimination of the roots of the quintic, when its coefficients are real, is given in terms of the two absolute invariants spoken of. In $\$ 3$, a proof of the results of $\S \mathbf{2}$ is given, by means of Sylvester's canonical form. The roots of the quintic were first discriminated in terms of the invariants by Hermite (Camb. and Dublin Math. Jour., Vol. Ix.); much simpler results were obtained by Sylvester (Phil. Trans., Vol. curv., 1864 ; Compt. Rend., Vol. LIx., 1864), and these have been partly reconsidered, and the results, as stated by Sylvester in the first of these papers, corrected by Salmon, Higher Algebra, and Cayley, Coll. Papers, Vol. vi. In general the method followed here, in $\$ 3$, is put together from Sylvester's memoir; but for the case when the quintic has a repeated root, which is not considered by Salmon or Cayley, I have made an independent examination.

1.

Let $I, J, K, L$ be the invariants, respectively, of degrees $18,4,8,12$, belonging to a binary quintic form, given for example in Salmon's Higher Algebra, 1885, Lesson xviII.; they are connected by the equation

$$
16 I^{2}=J\left(K^{2}-J L\right)^{2}+8 K^{3} L-72 J K L^{2}-432 L^{8} .
$$


Put

$$
\xi=\frac{L^{4}}{\left(K^{2}-J L\right)^{8}}, \quad \eta=\frac{K L^{2}}{\left(K^{2}-J L\right)^{2}}, \quad \xi=\frac{I}{K^{2}-J L},
$$

so that $\xi, \eta, \zeta$ are invariants respectively of degrees 0,0 and 2 ; also let $\varpi$ denote the absolute invariant, rational in $\hat{\xi}$ and $\eta$, given by

then we have

$$
\varpi=-\xi+\eta^{2}+72 \xi \eta-492 \xi^{2}-64 \eta^{3} ;
$$

$$
\begin{aligned}
\varpi\left(K^{2}-J L\right)^{6} & =-L^{4}\left(K^{2}-J L\right)^{3}+K^{2} L^{4}\left(K^{2}-J L\right)^{2}+72 K L^{6}\left(K^{2}-J L\right) \\
& -432 L^{8}-64 K^{9} L^{6} \\
& =L^{5}\left\{J\left(K^{2}-J L\right)^{2}+8 K^{3} L-72 J K L^{2}-432 L^{3}\right. \\
& =16 I^{2} L^{5}
\end{aligned}
$$

so that $\quad \varpi=\frac{16 I^{2} L^{5}}{\left(K^{2}-J L\right)^{6}}$;

hence

$$
\frac{2^{16} \xi^{5} \xi^{9}}{\varpi^{4}}=\frac{2^{16} L^{20} I^{9}}{\left(K^{2}-J L\right)^{24}} \frac{\left(K^{2}-J L\right)^{24}}{2^{16} I^{8} L^{20}}=I,
$$

$$
\begin{gathered}
\frac{2^{4}\left(\eta^{2}-\hat{\xi}\right) \zeta^{c^{2}}}{\varpi}=\frac{2^{4}\left[K^{2} L^{4}-L^{4}\left(K^{2}-J L\right)\right] I^{2}}{\left(K^{2}-J L\right)^{6}} \frac{\left(K^{2}-J L\right)^{6}}{2^{4} I^{2} L^{5}}=J, \\
\frac{2^{8} \xi^{2} \eta \xi^{4}}{\varpi^{2}}=\frac{2^{8} L^{8} K L^{2} I^{4}}{\left(K^{2}-J L\right)^{12}} \frac{\left(K^{2}-J L\right)^{12}}{2^{8} I^{4} L^{10}}=K, \\
\frac{2^{12} \xi^{4} \xi}{\varpi^{3}}=\frac{2^{12} L^{16} I^{6}}{\left(K^{2}-J L\right)^{18}} \frac{\left(K^{2}-J L\right)^{18}}{2^{12} I^{6} L^{15}}=L,
\end{gathered}
$$

that is $I=\frac{2^{16} \xi^{5} \xi^{9}}{\varpi^{4}}, \quad J=\frac{2^{4}\left(\eta^{2}-\hat{\xi}\right) \xi^{2}}{\varpi}, \quad K=\frac{2^{8} \xi^{2} \eta^{-4}}{\varpi^{2}}, \quad L=\frac{2^{12} \xi^{4} \xi^{6}}{\varpi^{3}}$,

whereby the four invariants $I, J, K, L$ are rationally expressed in terms of the three invariants $\xi, \eta, \xi$, which are themselves rational in $I, J, K, L$.

2.

We put

$$
X=12^{3} \cdot \hat{\xi}, \quad Y=48 \cdot \eta,
$$

and regard $X, Y$ as rectangular Cartesian coordinates. The discriminant $D$, of the quintic, known to be equal to $J^{2}-2^{7} K$, is given by

$$
\begin{aligned}
D & =\frac{2^{\S} \xi^{4}}{\varpi^{2}}\left[\left(\eta^{2}-\xi\right)^{2}-2^{7} \xi^{2} \eta\right] \\
& =\frac{\xi^{4}}{2^{4} \cdot 3^{6} \varpi^{2}}\left[\left(\frac{3}{4} Y^{2}-X\right)^{2}-\frac{\kappa}{3} X^{2} Y\right] .
\end{aligned}
$$


The curve

$$
\left(\frac{3}{4} Y^{2}-X\right)^{2}=\frac{8}{3} X^{2} Y
$$

has a cusp at the origin, one cuspidal branch lying below the asymptote $Y=\frac{3}{8}$, the other cutting it, either of these being continuous at infinity with a branch lying above the asymptote for every point of which $X \gtrless-1$; this is the curve against which the letter $D$ is placed in the diagram.

The curve $\varpi=0$, easily seen to be the same as

$$
(X-3 Y+2)^{2}+4(Y-1)^{3}=0,
$$

touches the curve $D$ at $\left[-2\left(\frac{6}{7}\right)^{3}, \frac{24}{45}\right]$, and cuts it at $\left(2^{8} / 11^{3}, 3.2^{5} / 11^{2}\right)$, and for the present need only be drawn between these points.

Thereby the tinite portion of the plane may he said to be divided into four regions. The quintic being supposed to have real coefticients, each of these regions corresponds to a definite number of real roots of the quintic; these numbers are placed in the diagram.

Further, the curve $D$ is, by the arc of $\varpi=0$ which is drawn, divided. we may say, into four arcs; each of these corresponds to a definite number of real roots of the quintic, shewn by the attached numbers; and the points of the arc of $\varpi=0$ which is drawn correspond to one or other of two cases, in regard to the number of real roots, according as $L \gtreqless 0$.

Finally, the origin, and the two points spoken of, where $\varpi=0$ meets the curve $D$, are associated with certain numbers of real roots of the quintic. These are given in association with the diagram.

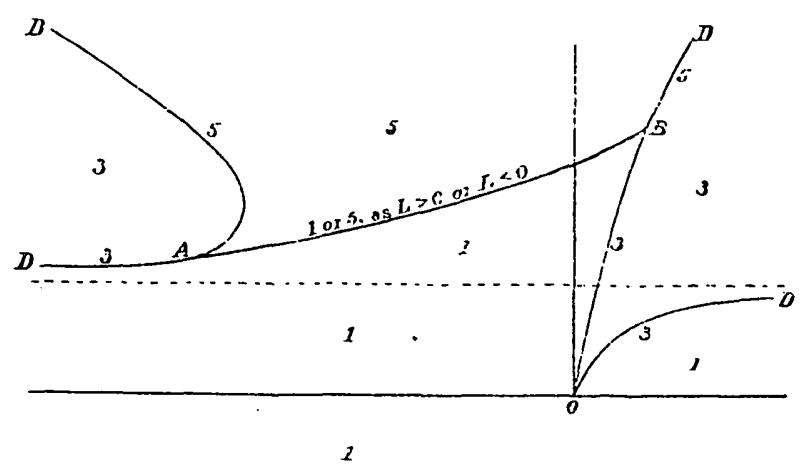

At $A$, if $L>0$, there is 1 real root and two complex roots both repeated : if $L<0$, there is 1 real root and two real roots both repeated, 5 in all.

At $B$, if $L>0$, there are 3 real roots, one a double root;

if $L<0$, there are 5 real roots, one a double root. 
1907.] INvariants of a binary quintid and the Realitty of its Roots. 125

At 0 , if $D>0$, there is 1 real root;

if $D \gtrless 0$, there are 3 real roots.

Analytically,

$D<0,3$ real roots.

$D>0, \varpi<0, Y>24 / 49,5$ real roots.

$D>0$, $\varpi>0$, or $Y<24 / 49$, or both, 1 real root.

$D=0, \varpi<0, Y>24 / 49,5$ real roots.

$D=0$, $\varpi>0$, or $Y<24 / 49$, or both, 3 real roots.

$D=0, \varpi=0, Y=24 / 49, L>0,1$ real root; $L<0,5$ real roots.

$D=0, \varpi=0, Y=3.2^{5} / 11^{2}, L>0,3$ real roots; $L<0,5$ real roots.

$\left.\begin{array}{l}D>0, \varpi=0, Y=0,1 \text { real root } \\ D \leqslant 0, \varpi=0, Y=0,3 \text { real roots }\end{array}\right\}$ included in a former statement.

3.

We consider the quintic with real coefficients

$$
a x^{5}+5 b x^{4} y+10 c x^{3} y^{2}+10 d x^{2} y^{3}+5 e x y y^{4}+f y^{5},
$$

excluding from consideration the case when three or more roots are equal.

We assume, then, that the quintic can be brought to the form

$$
r u^{5}+s v^{5}+t w w^{5}
$$

where $u, v, w$ are linear functions of $x$ and $y$, chosen so that their sum is zero, and $r, s, t$ are constants, provided that the invariant $L$ does not vanish; and that, if $L$ vanishes, and no other restrictive condition is satisfied, the quintic can be brought to the form

$$
A u^{5}+5 E u v^{4}+F v^{5},
$$

where $u, v$ are real linear forms, and $A, E, F$ are real constants, while if $L=0, J=0$, this form reduces to

$$
A u^{5}+5 E u v^{4}
$$

and that, in all other cases in which $L=0$, save those in which the quintic has three or more equal roots, the first form

$$
r u^{5}+s v^{5}+t w^{5}
$$

is valid (Sylvester, loc. cit.). The invariants $J, K$ of the form

$$
A u^{5}+5 E u v^{4}+F v^{5}
$$

wre $J=A^{2} F^{2}, K=-2 A^{3} E^{5}$ (Salmon, Higher Algebra). 
We put

$$
\hat{\xi}=\frac{L^{4}}{\left(K^{2}-J L\right)^{3}}, \quad \eta=\frac{K L^{2}}{\left(K^{2}-J L\right)^{2}},
$$

and interpret

$$
X=12^{3} \xi, \quad Y=48 \eta
$$

as rectangular Cartesian ccordinates. The cases $L=0$, or $L=0$, $J=0$, lead then to the single point $X=0, Y=0$, which we consider, briefly, later; for all other cases we may deal with the quintic under the form $r u^{5}+s v^{5}+t w^{5}$.

Calculated for this form the invariants of the quintic are

$$
\begin{gathered}
I=r^{5} s^{5} t^{5}(s-t)(t-r)(r-s), \quad K=r^{2} s^{2} t^{2}(s t+t r+r s), \quad L=r^{4} s^{4} t^{4}, \\
J=(s t+t r+r s)^{2}-4 r s t(r+s+t) ;
\end{gathered}
$$

these give, if $\mu=4(r+s+t)$,

$$
\frac{1}{\mu^{2}}\left(s t+t r+r s=\eta, \quad \frac{1}{\mu^{4}} r s t(r+t+s)=\frac{1}{4} \xi, \quad \frac{1}{\mu^{6}} r^{2} s^{2} t^{2}=\xi^{2},\right.
$$

so that the three quantities $\mu^{-2} s t, \mu^{-2} t r, \mu^{-2}$ rs are the roots of the cubic equation in $\psi$,

$$
\psi^{3}-\eta \psi^{2}+\frac{1}{4} \xi \psi-\xi^{2}=0
$$

the discriminant of this is at once found to be

$$
-\xi^{2}\left(-\xi+\eta^{2}+72 \xi \eta-432 \xi^{2}-64 \eta^{3}\right),
$$

or

$$
-\xi^{2} \varpi
$$

so that the roots of the cubic are real when $\varpi$ is positive, and two of them conjugate imaginaries when $\varpi$ is negative. We have found

$$
\varpi / L=16 I^{2} L^{4} /\left(K^{2}-J L\right)^{6},
$$

so that the signs of $L$ and are the same; thus, as $L$ is a negative multiple of the discriminant of the canonizant

$$
\left|\begin{array}{cccc}
a & b & c & d \\
b & c & d & e \\
c & d & e & f \\
y^{3} & -y^{2} x & y x^{2} & -x^{3}
\end{array}\right|,
$$

of which $u, v, w$ are the linear factors,

(1) When $\varpi$ is positive, in the canonical form

$u, v, v, r, s, t$ are real ;

$$
r u^{5}+s v^{5}+t w^{5}=0
$$

(2) When $\varpi$ is negative, two of the linear forms $u, v, w$, which we take to be $u$ and $v$, are conjugate imaginaries, and $w$ is real, while, correspondingly, $r$ and $s$ are conjugate imaginaries and $t$ is real. Taking account 
of the fact that the original quintic is real, these results are easily found to hold also when $t=0$, in which case both $L$ and the canonizant vanish identically.

Conversely, when $\xi$ and $\eta$ are assigned, the above cubic determines ratios for the coefficients $r, s, t$, and, when $\varpi$, which is a definite function of $\xi, \eta$, is positive, we may take real linear functions $u, v, v$, while, when $\varpi$ is negative, we may take $u, v$ conjugate complex linear functions ; thus, save when $\varpi=0$, to each value of $\xi$ and $\eta$ belongs a class of quintic forms. When $\sigma=0$, and $L \neq 0$, we may take $r=s$, both these being real since they are roots of a cubic equation with real coefficients, but the linear forms $u, v$ may be either real or conjugate complexes.

Consider now the discriminant, $D$, of the quintic; it is known that, when it is negative, the quintic has three real roots, and, when it is positive, the quintic has either one or five real roots; its value is

$$
\begin{aligned}
D & =J^{2}-2^{7} K \\
& =\frac{2^{8} \xi^{4}}{\varpi^{2}}\left[\left(\eta^{2}-\hat{\xi}\right)^{2}-2^{7} \hat{\xi}^{2} \eta\right],
\end{aligned}
$$

where, as before, $\quad \zeta=\frac{I}{K^{2}-J L}$;

thus $D$ is positive or negative according as $\left(\eta^{2}-\xi\right)^{2} \gtreqless 2^{7} \xi^{2} \eta$, or, substituting $\hat{\xi}=12^{-3} X, \eta=48^{-1} Y$, according as

$$
\left(\frac{3}{4} Y^{2}-X\right)^{2} \gtrless \frac{8}{3} X^{2} Y
$$

namely, $\quad\left(1-\frac{8}{3} Y\right)\left\{X-\frac{\frac{3}{4} Y^{2}}{1+\left(\frac{8 Y}{3}\right)^{3}}\right\}\left\{X-\frac{\frac{3}{4} Y^{2}}{1-\left(\frac{8 Y}{3}\right)^{3}}\right\} \geq 0$.

The curve $D=0$ is thus as in the figure

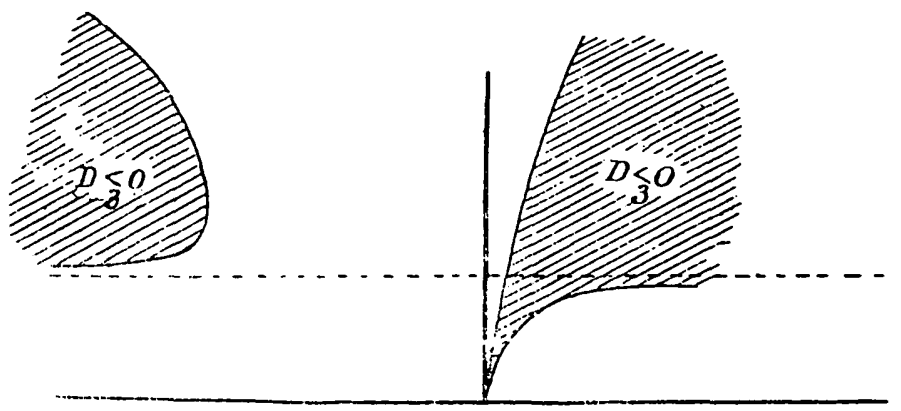

having a cusp at the origin, the line $Y=\frac{3}{8}$ for an asymptote, cutting the 
asymptote at $\left(3^{3} .2^{-9}, 3.2^{-3}\right)$, or $\left(\cdot 05,3.2^{-3}\right)$, and having a vertical tangent at $\left(-1, \frac{2}{3}\right)$. The portion of the plane for which $D$ is negative, and the quintic has three real roots, is that shaded.

Consider next the function $\boldsymbol{\sigma}$; putting

$$
\xi=12^{-3} X, \quad \eta=48^{-1} Y,
$$

we have

$$
\begin{aligned}
\varpi & =-\xi+\eta^{2}+72 \hat{\xi} \eta-432 \xi^{2}-64 \eta^{3} \\
& =-\frac{1}{3^{3} \cdot 4^{4}}\left[4 X-3 Y^{2}-6 X Y+X^{2}+4 Y^{3}\right] \\
& =-\frac{1}{3^{3} \cdot 4^{4}}\left[(X-3 Y+2)^{2}+4(Y-1)^{3}\right] .
\end{aligned}
$$

Thus the curve $\varpi=0$, like $D=0$, is unicursal, satisfied, for instance, by taking

$\tau=\frac{4}{5} \frac{31 . Y-7 . X-24}{X-Y}, \quad X=3^{3} .2^{8} \frac{5 \tau-4}{(5 \tau+28)^{3}}, \quad Y=3.2^{5} \frac{5 \tau+4}{(5 \tau+28)^{2}}$,

where the parameter $\tau$ is chosen for its simplicity in a subsequent application. The curve is as in the figure

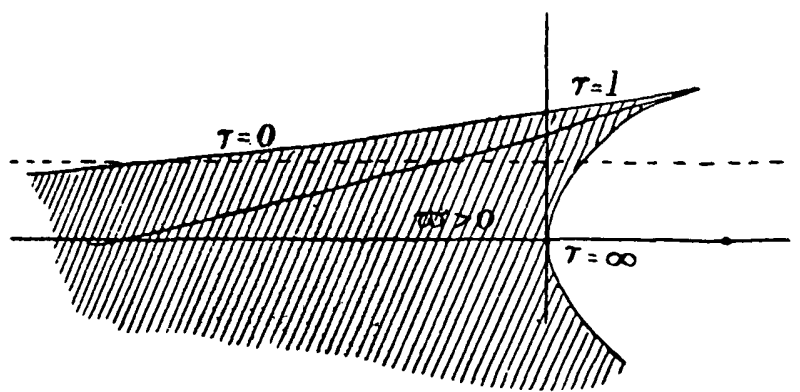

having a cusp at $X=1, Y=1$, whose tangent passes through $(-2,0)$, one side of the curve cutting $Y=\frac{3}{8}$ in $\left(-1 \cdot 86,3.2^{-3}\right)$ and $Y=0$ in $(-4,0)$, while the other side touches $X=0$ at the origin and cuts $Y=\frac{3}{8}$ in ('11, 3.2 $\left.2^{-3}\right)$, that is, to the right of the point in which $D=0$ cuts $Y=\frac{3}{8}$; in fact, this side of $\varpi=0$ lies, so long as $Y$ is positive, inside the shaded portion of $D=0$ for which $X$ is positive. Putting $\tau=1$ in the formulæ above, we obtain

$$
X=2^{8} / 11^{3}, \quad Y=3.2^{5} / 11^{2},
$$

which is a point also on the curve $D=0$, obtained by taking, in the formulæ

$$
X=3^{3} \cdot 2^{-8} \cdot \mu^{4} /(\mu+1), \quad Y=3.2^{-3} \cdot \mu^{2},
$$

which give a parametric representation of $D=0$, the value $\mu=2^{4} / 11$; 
putting, in the above formulæ for $\varpi=0$, the parameter $\tau=0$, we obtain

$$
X=-2\left(\frac{6}{7}\right)^{3}, \quad Y=\frac{24}{49}, \quad \frac{d Y}{d X}=\frac{7}{36},
$$

which are also obtained by taking $\mu=-\frac{8}{7}$ in the formulæ just put down for $D=0$; the curve $\varpi=0$ thus touches $D=0$ at this point. Finally, $\varpi$ is positive in the shaded region of the diagram between the two sides of the curve.

There is another curve which it is useful to trace for the sake of comparison with Sylvester's results, though it is not necessary for our purpose. We have

$$
2^{11} L-J^{3}=-\frac{2^{12} \xi^{6}}{12^{9} \varpi^{3}}\left[\left(\frac{3}{4} Y^{2}-X\right)^{3}-\frac{2^{5}}{3^{3}} X^{4}\right] ;
$$

the curve $2^{11} L-J^{3}=0$ is thus given by

$$
\frac{3}{4} Y^{2}=X\left(1+\frac{2^{\frac{5}{3}}}{3} X^{\frac{1}{3}}\right),
$$

or by

$$
\begin{gathered}
X=3^{3} \cdot 2^{-5}\left(\sigma^{2}-1\right)^{-3}, \quad Y=3.2^{-\frac{3}{3}} \sigma\left(\sigma^{2}-1\right)^{-2}, \\
\sigma=3^{2} \cdot 2^{-\frac{3}{3}} \frac{Y}{X}\left(\frac{3}{4} \frac{Y^{2}}{X}-1\right) ;
\end{gathered}
$$

the curve has therefore such a shape as given in the figure

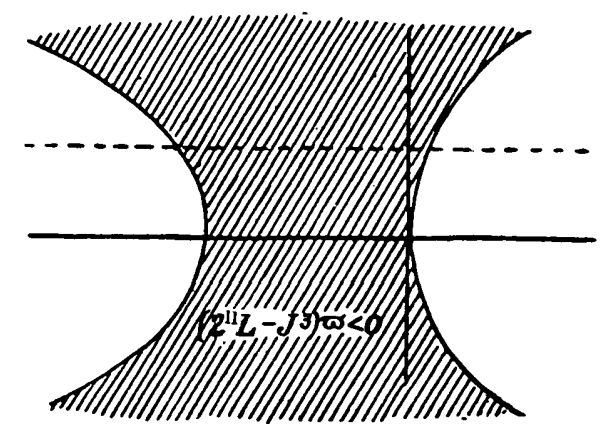

cutting $Y=0$ in $\left(-\frac{27}{3}, 0\right)$ and $(0,0)$, and cutting $Y=\frac{3}{8}$ in, approximately, $\left(-1, \frac{3}{8}\right)$ and $\left(\cdot 065, \frac{3}{8}\right)$, so that for positive $X$ the right hand branch of the curve lies to the right of the branch $X=\frac{3}{4} Y^{2} /\left[1+\left(\frac{8}{3} Y\right)^{\frac{1}{2}}\right]$ of $D=0$, and to the left of the portion of $\varpi=0$ which is on the right of the cuspidal tangent of this. It is important to notice that the curve passes through the point of contact of $D=0$ and $\varpi=0$, namely,

$$
X=-2.6^{3} .7^{-3}, \quad Y=24.7^{-2} .
$$

The portion of the plane for which $\left(2^{11} L-J^{3}\right) \varpi<0$ is that shaded in the figure; for part of this $\varpi$ is positive and for part of it negative. 
Denoting $2^{11} L-J^{3}$ by $\Lambda$, the relations of the three curves are shewn in the following diagram, which is drawn, however, with only approximate accuracy.

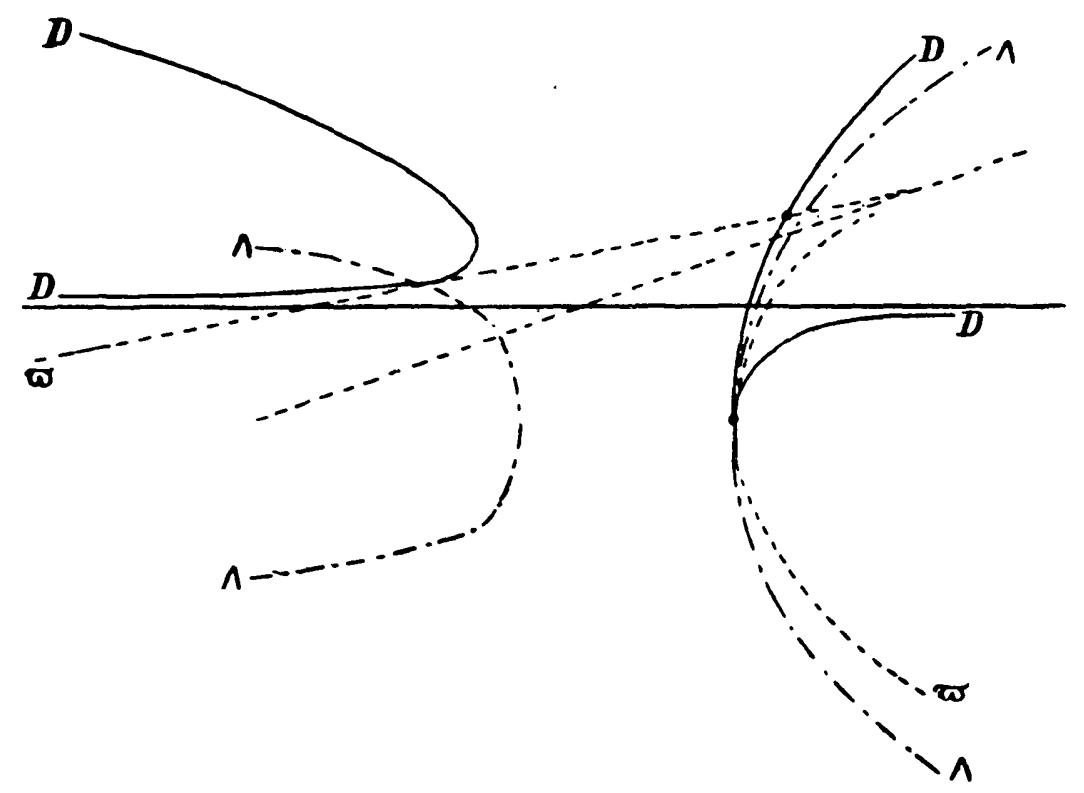

Consider now the equation

$$
r u^{5}+s v^{5}-t(u+v)^{5}=0,
$$

when $\varpi=0$, so that we may take $r=s$. Then, beside the root given by $u+v=0$, we have

$$
\frac{u^{4}-u^{8} v+u^{2} v^{2}-u v^{3}+v^{4}}{u^{4}+4 u^{8} v+6 u^{2} v^{2}+4 u v^{3}+v^{4}}=\frac{t}{r} ;
$$

the left side is a function of $\theta=\frac{u^{2}+v^{2}}{u v}$,

and the equation gives, if $\quad \tau=\frac{4}{5}\left(1+\frac{4 t}{r}\right)$,

the values expressed by $\quad \theta=\frac{-2 \sqrt{ } \tau}{\sqrt{ } \tau \mp 2}$,

and thence, if $\epsilon^{2}=1, \xi^{2}=1$, the four values for $u / v$ expressed by

$$
\frac{u}{v}=\frac{1+\xi(\epsilon \sqrt{ } \tau-1)^{\frac{1}{2}}}{1-\xi(\epsilon \sqrt{ } \tau-1)^{\frac{1}{2}}}
$$


n terms of $r, s, t$, taking account of $r=s$, the invariants of the quintic are

$$
\begin{gathered}
J=\left(r^{2}+2 t r\right)^{2}-4 r^{2} t(2 r+t), \quad K=r^{4} t^{2}\left(r^{2}+2 r t\right), \quad L=r^{8} t^{4}, \\
K^{2}-J L=4 r^{10} t^{5}(2 r+t),
\end{gathered}
$$

from which $X, Y$ are expressible in terms of $\tau$; the result is that parametric expression for $\varpi=0$ which was given above; we can thus obtain, as we know the values of this parameter for the various portions of this curve, the forms of the four values of $u / v$ for the various portions.

Thus, for the portion of $\varpi=0$ for which $X>0, Y<0$, and the portion for which $X<-2.6^{3} .7^{-3}$, through which the parameter

$$
\tau=\frac{4}{3} \frac{31 \cdot Y-7 \cdot X-24}{X-Y}
$$

remains negative, constantly increasing from $-\infty$ to 0 , we have, if $\omega$ be a real positive quantity, and $\tan \theta=-\epsilon \omega$,

$$
\begin{aligned}
\frac{u}{v} & =\frac{1+\xi(\kappa i \omega-1)^{\frac{1}{3}}}{1-\zeta(\epsilon i \omega-1)^{\frac{1}{2}}} \\
& =\frac{1+\xi\left(1+\omega^{2}\right)^{\frac{1}{2}} \cos \frac{1}{2} \theta+i \xi\left(1+\omega^{2}\right)^{\frac{1}{2}} \sin \frac{1}{2} \theta}{1-\xi\left(1+\omega^{2}\right)^{\frac{1}{2}} \cos \frac{1}{2} \theta-i \zeta^{\xi}\left(1+\omega^{2}\right)^{\frac{1}{2}} \sin \frac{1}{2} \theta},
\end{aligned}
$$

and this is always a complex quantity of modulus other than unity (save for the final point $\cos \frac{1}{2} \theta=0, \tau=0$ ). Therefore, if $L$ be positive, in which case $u, v$ are real linear functions of the original variables $x, y$ of the quintic equation, the quintic has only one real root, given by $u=-v$; while, if $L$ be negative, in which case $u, v$ are conjugate complex linear functions and $u / v$ is of modulus unity, there is still only one real root, that given by $u=-v$. Next, for the portion of $\varpi=0$ lying between the two branches of $D=0$, for which $X$ varies from $-2.6^{3} \cdot 7^{-3}$ to $2^{8} \cdot 11^{-3}$, and $\tau$ varies from 0 to 1 , each of the four numbers expressed by

$$
\left[1+\xi(\epsilon \sqrt{ } \tau-1)^{\frac{1}{2}}\right] /\left[1-\xi(\epsilon \sqrt{ } \tau-1)^{\ddagger}\right]
$$

is complex, but of modulus unity ; thus, when $L>0$, the quintic has four complex roots corresponding to these, and one real root given by $u+v=0$, while, when $L<0$, the quintic has five real roots. Lastly, for the portion of $\varpi=0$ lying between the cuspidal branches of $D=0$ for which $X>0, Y>0$, the value of $\tau$ increases constantly from 1 to $\infty$; thus, for $\epsilon=1$, the two corresponding numerical values of $u / v$ are real, while those belonging to $\epsilon=-1$ are complex of modulus unity; wherefore, when $L>0$, the quintic has three real roots, including that given 
by $u+v=0$, and the same is true when $L<0$; this result only verifies a previously obtained result, for along this portion of the curve, as we have seen, we have $D<0$. These results are summarised in the diagram

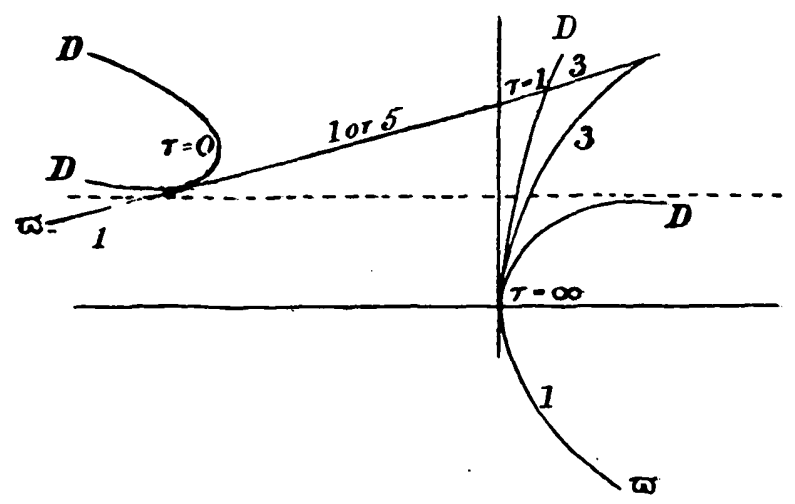

We have already remarked that to any point $(X, Y)$ not upon $\varpi=0$, corresponds first a definite set of ratios for the coefficients $r, s, t$, and then, either three real linear forms $u, v, w$, or three of the form $-\frac{1}{2}(w+i \sigma),-\frac{1}{2}(w-i \sigma), w$, where $w, \sigma$ are real, according as $\sigma>0$ or $\varpi<0$; and it is clear that all equations of the form

$$
f(x, y)=r(\lambda x+\mu y)^{5}+s\left(\lambda^{\prime} x+\mu^{\prime} y\right)^{5}-t\left[\left(\lambda+\lambda^{\prime}\right) x+\left(\mu+\mu^{\prime}\right) y\right]^{5}=0,
$$

in which $r, s, t$ are given, have the same number of real roots whatever the values of the real coefficients $\lambda, \mu, \lambda^{\prime}, \mu^{\prime}$; as also have all equations of the form

$$
\begin{gathered}
F(x, y)=r\left[\lambda x+\mu y+i\left(\lambda^{\prime} \dot{x}+\mu^{\prime} y\right)\right]^{5}+s\left[\lambda x+\mu y-i\left(\lambda^{\prime} x+\mu^{\prime} y\right)\right]^{5} \\
-t[2 \lambda x+2 \mu y]^{5}=0 .
\end{gathered}
$$

But further, still supposing $\varpi \neq 0$, and supposing also now that $D \neq 0$, this number will be unaffected by variation of $X, Y$, that is, of $r, s, t$, provided the variation be sufficiently limited in extent. For, putting $y=1$ ind $t=1$, let $x$ be a root of one of these equations, and $x+\delta x$ the root correspondingly belonging to the values $r+\delta r, s+\delta s$; we then have, in the two cases,

$$
5\left(r \lambda u^{4}+s \lambda^{\prime} v^{4}\right) \delta x+\delta r \cdot u^{5}+\delta s . v^{5}=0,
$$

or

$$
5\left[r \cdot\left(\lambda+i \lambda^{\prime}\right) u^{4}+s\left(\lambda-i \lambda^{\prime}\right) v^{4}\right] \delta x+\delta r \cdot u^{5}+\delta s . v^{5}=0,
$$

shewing that $\delta x$ is real when $x$ is real, and complex (including real) when $x$ is complex. The inference, however, holds equally well when $r, s, t$ corre- 
spond to a point upon $\varpi=0$, provided that the variation of $r, s, t$ is such as to preserve the forms of $u, v, w$, that is, such as to preserve the sign of $L$.

Now, for the portions of $\varpi=0$ corresponding to $-\infty<\tau<0$, we have seen that there is one real root, whether we have $L>0$ or $L<0$ : there is thus one real root for the regions of the plane on either side of these portions of $\boldsymbol{\sigma}=0$, the limitation of these regions of the plane being only parts of the curves $D=0$ or $\varpi=0$. There are similarly three real roots on either side of the portion of the curve $\varpi=0$ for which $1<\tau<x$, a fact which we have proved independently. For the portion of $\varpi=0$ for which $0<\tau<1$, we have proved that there is one or five real roots according as $L>0$ or $L<0$; when therefore we pass to the side of this portion of $\varpi=0$ for which $\varpi,=16 I^{2} L^{5} /\left(K^{2}-J L\right)^{6}$, is positive, and therefore $L$ positive, we shall move into a region for which there is one real root, and when we pass to the side of this portiont of $\varpi=0$ for which $\varpi<0$ or $L<0$, there will be five real roots; this is the region, in the figure, above $\varpi=0$ and between the branches of $D=0$. We therefore have the diagram

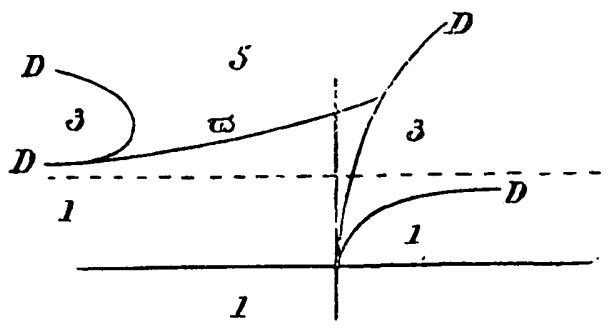

the only separations of regions being the curve $D=0$ and the portion of $\varpi=0$ for which $0<\tau<1$. Talking account of the behaviour of the curve $\Lambda=0$, the results may be stated analytically by saying that if $D<0$, there are three real roots, while if $D>0$, there are five real roots when both $L<0$ and $\Lambda>0$, but only one real root if either or both of $L>0, \Lambda<0$. This is the result as stated by Salmon, correcting the form given by Sylvester in his paper in the Phil. Trans., which, however, does not agree with the result of his own arguments. If an analytical statement is required, it is more natural from the present point of view to say that, when $D>0$, there are five real roots, when $L$ (or $\varpi)<0$ and $Y>\frac{24}{45}$, but only one real root if $L>0$, or $Y<\frac{2}{49}$, or both ; the condition $Y>\frac{24}{49}$ is the same as $98 K L^{2}>\left(K^{2}-J L\right)^{2}$. Our diagrams show at once the reason for the indeterminate form which Sylvester was able to give to one of his criteria-the curve $\Lambda=0$ only intervenes in the 
analytic statement in order to divide the curve $\varpi=0$ into portions.

A part verification of the preceding results is worth noticing: suppose $D>0$, so that the number of real roots is 5 or 1 ; suppose further that $\varpi>0$, so that in the form

$$
r u u^{5}+s v^{5}-t(u+v)^{5}=0,
$$

all of $r, s, t, u, v$ are real ; if this equation had $\check{b}$ real roots its derivative in regard to $u$, or

$$
r \cdot u^{4}-t(u+v)^{4}=0,
$$

would have 4 ; it has however at most 2 , and this only when $r$ and $t$ are of the same sign. Thus. for $D>0, \varpi>0$, the quintic has only one real root. We have seen that it has only one real root also in some cases in which these conditions are not satisfied.

We examine now equations for which $D=0$. When the equation

$$
r u u^{5}+s v^{5}-t(u+v)^{5}=0
$$

has the repeated root $u / v=\theta$, this is also a root of the two derivatives

$$
r u^{4}-t(u+v)^{4}=0, \quad s v^{4}-t(u+v)^{4}=0 .
$$

and the original equation may be written

$$
\left(\frac{\theta+1}{\theta}\right)^{4} u^{5}+(\theta+1)^{4} v^{\bar{n}}-(u+\imath)^{\bar{j}}=0 ;
$$

the left side of this, multiplied by $\theta$, is

$$
(u-v \theta)^{2}\left(A \theta^{-3} u^{3}+B \theta^{-2} u^{2} v+C^{\prime} \theta^{-1} u v^{2}+D v^{3}\right),
$$

where

$$
\begin{aligned}
& A=4 \theta^{3}+6 \theta^{2}+4 \theta+1, \\
& B=3 \theta^{3}+12 \theta^{2}+8 \theta+2, \\
& C=2 \theta^{3}+8 \theta^{2}+12 \theta+3, \\
& D=\theta^{3}+4 \theta^{2}+6 \theta+4,
\end{aligned}
$$

and the discriminant of the cubic factor of this, in regrard to $u / v$, save for a positive numerical factor, is found to be

$$
\left(\frac{\theta+1}{\theta}\right)^{6}\left(\theta^{2}+1\right)\left(\theta^{2}+2 \theta+2\right)\left(2 \theta^{2}+2 \theta+1\right) .
$$

Now, unless the original quintic have two pairs of equal roots which are 
conjugate imaginaries, a case which will be proved to correspond only to the single point $X=-2.6^{3} .7^{-3}, Y=24.7^{-2}$, the repeated root of the quintic will be real ; thus when $L>0$, and $u, v$ are real, $\theta$ is real, and when $L<0$, and $u, v$ are conjugate imaginaries, $\theta$ is of the form $e^{i \beta}$. In the latter case, if $u=-\frac{1}{2}(v+i \sigma), v=-\frac{1}{2}(v-i \sigma)$, the discriminant of the cubic in regard to the real quantities $w, \sigma$ will differ from that just put down by a factor

$$
\left[\frac{\partial(u, v)}{\partial(w, \sigma)}\right]^{\frac{1}{2} \cdot 3 \cdot 4}=-\frac{1}{64}
$$

and may be taken to be the negative of that; putting, when $\theta=e^{i \beta}$, $\cos \beta=t$, the discriminant of the cubic in regard to the real quantities $w, \sigma$ may thus be found to be, save for a positive numerical factor,

$$
-(1+t)^{3} t\left(5+12 t+8 t^{2}\right) ;
$$

the sign of this is therefore the sign of $-t$, while the sign of the former discriminant, for $\theta$ real, is always positive.

Next, putting $r=(\theta+1)^{4} / \theta^{4}, s=(\theta+1)^{4}, t=1$, and calculating the invariants $J, K, L$ of the quintic, and hence the values of $X$ and $Y$, we find

$$
X=\frac{3^{3} \theta^{8}(\theta+1)^{8}}{\left[(\theta+1)^{4}+\theta^{4}+\theta^{4}(\theta+1)^{4}\right]^{3}}, \quad Y=\frac{3 \theta^{4}(\theta+1)^{4}\left[\theta^{4}+(\theta+1)^{4}+1\right]}{\left[(\theta+1)^{4}+\theta^{4}+\theta^{4}(\theta+1)^{4}\right]^{2}},
$$

which, putting $\quad 1-\frac{4 X}{3 Y^{2}}=\frac{\mu}{\mu+1}=\frac{4 \theta^{2}(\theta+1)^{2}}{\left(\theta^{2}+\theta+1\right)^{3}}$,

lead to

$$
X=\frac{3^{3} \cdot 2^{-\mathrm{s}} \cdot \mu^{4}}{1+\mu}, \quad Y=\frac{3}{8} \mu^{2},
$$

which is the previously obtained parametric expression of the curve $D=0$; to each point of this curve there belong the six values of $\theta$ given by

$$
\theta, \quad \frac{1}{\theta},-(1+\theta), \quad-\frac{1}{1+\theta},-\frac{\theta+1}{\theta},-\frac{\theta}{\theta+1},
$$

which correspond to the six ways in which we may permute the coefficients $r, s, t$ in the quintic. When $\theta=e^{i \beta}$, the expressions for $X, Y$ in terms of $t,=\cos \beta$, are

$$
X=\frac{3^{3} \cdot 2^{4}(t+1)^{4}}{\left(8 t^{3}+12 t^{2}-2 t-7\right)^{3}(2 t+1)^{3}}, \quad Y=\frac{3 \cdot 2^{3}(t+1)^{2}}{\left(8 t^{3}+12 t^{2}-2 t-7\right)^{2}} .
$$


Now, if $\theta$ is real and $0<\theta<1$, we have

$$
\begin{gathered}
-\infty<-\left(1+\frac{1}{\theta}\right)<-2<-(1+\theta)<-1<-\frac{1}{1+\theta} \\
<-\frac{1}{2}<-\frac{\theta}{1+\theta}<0, \\
1<\frac{1}{\theta}<\infty ;
\end{gathered}
$$

thus, in the equation $\quad \frac{4 \theta^{2}(\theta+1)^{2}}{\left(\theta^{2}+\theta+1\right)^{3}}=\frac{\mu}{\mu+1}$,

to obtain the points of the curve $D=0$ for which $\theta$ is real, it is sufficient to take $0<\theta<1$, and each point of the curve will only arise for one value of $\theta$; then $\mu$ will vary from 0 to $2^{4} / 11$ and $(X, Y)$ from $(0,0)$ to $\left(2^{8} / 11^{3}, 3.2^{5} / 11^{2}\right)$; we have in fact already seen that these are the extreme points of the arc of $D=0$ which lies where $\varpi>0$. On this arc the cubic obtained by dividing the quintic by $(u-v \theta)^{2}$ has a positive discriminant, and only one real root; the quintic has thus three real roots and two conjugate imaginary roots.

We have next, if $\theta=e^{i \beta}, \cos \beta=t$,

$$
\frac{4 \theta^{2}(\theta+1)^{2}}{\left(\theta^{2}+\theta+1\right)^{3}}=\frac{4\left(\theta^{3}+\theta^{-\frac{1}{2}}\right)^{2}}{\left(\theta+\theta^{-1}+1\right)^{3}}=\frac{8(1+t)}{(2 t+1)^{3}},
$$

and the equation

$$
\frac{(2 t+1)^{3}}{t+1}=\frac{\left(2 t_{0}+1\right)^{3}}{t_{0}+1}
$$

gives, beside $t=t_{0}$,

$$
\left[2 t+1+\frac{1}{2}\left(2 t_{0}+1\right)\right]^{2}=\frac{1}{4}\left(2 t_{0}+1\right)^{2} \frac{t_{0}-1}{t_{0}+1},
$$

of which, when $-1<t_{0}<1$, the roots are imaginary; to a value $t$ such that $-1<t<1$ correspond both $\theta=e^{i \beta}$ and $\theta=e^{-i \beta}=1 / \theta$. We shall thus obtain every point of the curve $D=0$ for which $\theta=e^{i \beta}$ by supposing $\beta$ to vary continuously from 0 to $\pi$.

The whole path of $\theta$, in its plane, corresponding to one description of the curve $D=0$, is thus given by the open curve $O A B C D E$

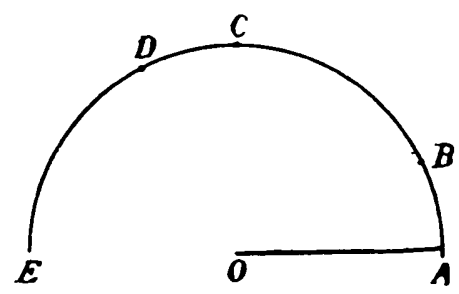


consisting of a radius $O A$ and a semicircle; as $\theta$ varies from 0 to $A$, that is, from 0 to 1 , the portion of $D=0$ is described lying between the origin and the point $\left(2^{8} .11^{-3}, 3.2^{5} \cdot 11^{-2}\right)$, where $D=0$ is cut by $\varpi=0$; along this arc the discriminant of the cubic factor is positive and the quintic has three real roots. As $\theta$ passes along the semicircle towards $\theta=i$, $t,=\cos \beta$, diminishes from 1 to a value (between 7 and $\cdot 68$ ) for which $(2 t+1)^{3}=8(t+1)$, for which $\mu$ is infinite and $(X, Y)=(\infty, \infty)$; the corresponding value of $\beta$ is denoted by the point $B$ of the semicircle; the point $(X, Y)$ of $D=0$, corresponding to the arc $A B$ of the senicircle, passes from the intersection of $D=0$ and $\varpi=0$, along the left hand cuspidal branch of $D=0$ for which $X>0$, to infinity; along this arc the discriminant

$$
-(1+t)^{9} t\left(5+12 t+8 t^{2}\right)
$$

is negative and the quintic has five real roots. As $\theta$ passes along the semicircle from $B$ to $C$, the fraction $8(t+1) /(2 t+1)^{3}$, or $\mu /(\mu+1)$, constantly increases from unity, being for $t=0$ equal to 8 , and $\mu$ is negative, passing from $-\infty$ to $-\frac{8}{7}$; thus $(X, Y)$ describes the branch of $D=0$ for which $X<0$, from $(\infty, \infty)$ to the point $\left(-2.6^{3} .7^{-3}, 24.7^{--1}\right)$ at which $D=0$ is touched by $\varpi=0$; along this portion the discriminant of the cubic is still negative and the quintic has five real roots. As $\theta$ passes along the semicircle from $C$ to $D$, where $\beta=\frac{2}{3} \pi$, the fraction $8(t+1) /(2 t+1)^{3}$ passes to infinity and $\mu$ passes to -1 ; the point $(X, Y)$ thus passes to $\left(-\infty, \frac{3}{8}\right)$; along this portion the discriminant of the cubic is positive and the quintic has three real roots. Finally, as $\beta$ passes from $\frac{2}{3} \pi$ to $\pi$, the fraction $\mu /(\mu+1)$ becomes negative, and $\mu$, which is negative, is numerically less than unity; thus $X$ is positive, and $(X, Y)$ passes from $\left(\infty, \frac{3}{8}\right)$ to the origin, corresponding to $t=-1$, or the point $E$ of the semicircle, along the portion of $D=0$ which is below the asymptote; for this branch the discriminant of the cubic remains positive and the quintic has three real roots. The results are thus those represented in the diagram

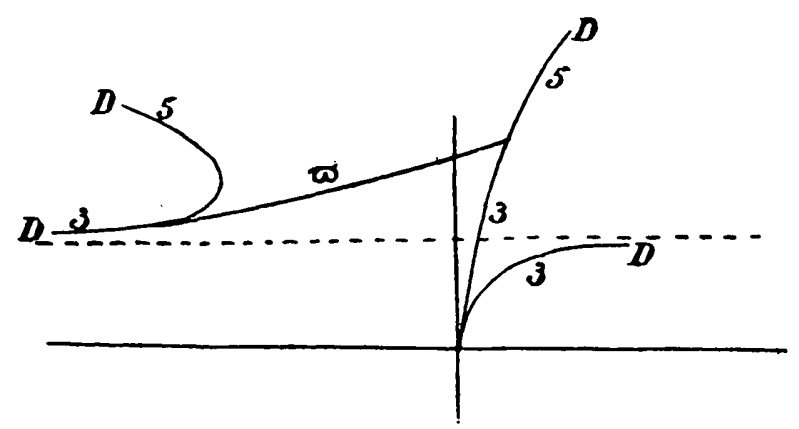


und, analytically, upon $D=0$, there are five real roots for $\varpi<0$, $Y>\frac{2 \pm}{4}$, but three real roots in all other cases; or otherwise, introducing Sylvester's eriterion $\Lambda=2^{11} L-J^{3}$, there are five real roots for $L<0$, $\Lambda>0$, and three in all other cases.

It remains now to examine the points of intersection of the curves $D=0, \varpi=0$.

At the point of contact $\left(-2.6^{3} \cdot 7^{-3}, 24.7^{-2}\right)$, regarded as lying on $\varpi=0$, we have seen that the values of $u / v$ are given, for $\tau=0$, by

that is, by

$$
\frac{u}{v}=-1, \quad \frac{u}{v}=\frac{1+\xi(\epsilon \sqrt{ } \tau-1)^{\frac{\xi}{3}}}{1-\xi(\epsilon \sqrt{ } \tau-1)^{\frac{1}{2}}}
$$

$$
\frac{u}{v}=-1, \quad \frac{u}{v}=\frac{1+i}{1-i}, \frac{1+i}{1-i}, \frac{1-i}{1+i}, \frac{1-i}{1+i},=i, i,-i,-i ;
$$

regarded as lying on $D=0$, we have found that the point corresponds to $\theta=i$, for which the discriminant

$$
\theta^{-6}(\theta+1)^{6}\left(\theta^{2}+1\right)\left(\theta^{2}+2 \theta+2\right)\left(2 \theta^{2}+2 \theta+1\right)
$$

vanishes; the other vanishing factors $\theta+i, \theta^{2}+2 \theta+2,2 \theta^{2}+2 \theta+1$ correspond in fact to the same point, arising by transforming the $\operatorname{root} \theta=i$ by

$$
\theta^{\prime}=\theta^{-1}, \quad \theta^{\prime}=-(\theta+1), \quad \ldots
$$

Thus, if $L>0$, and $u, v$ are real linear functions, there are two imaginary roots of the quintic, each repeated, and one real root given by $u+v=0$, while if $L<0$, and $u, v$ are conjugate linear functions, there are two real roots, each repeated, beside the real root given by

$$
u+v=0 \text {. }
$$

Conversely, if the original quintic bave the form

$$
10\left(c x_{1}+d y_{1}\right) x_{1}^{2} y_{1}^{2},
$$

proper to the case of two repeated roots, $x_{1}$ and $y_{1}$ being real or conjugate imaginary linear functions of the original variables $x, y$, the canonizant is

$$
\left(c x_{1}+d y_{1}\right)\left(c x_{1}+i d y_{1}\right)\left(c x_{1}-i d y_{1}\right)
$$

and, supposing neither $c$ nor $d$ to be zero, we have

$10\left(c x_{1}+d y_{1}\right) x_{1}^{2} y_{1}^{2}$

$=\frac{1}{2 c^{2} d^{2}}\left\{4\left[\frac{1}{2}(1-i)\left(c x_{1}+i d y_{1}\right)\right]^{5}+4\left[\frac{1}{2}(1+i)\left(c x_{1}-i c l y_{1}\right)\right]^{5}-\left[-c x_{1}-d y_{1}\right]^{5}\right\}$, 
from which we calculate, save for respective factors of the forms $\mu^{10}, \mu^{20}, \mu^{30}$,

$$
J=(16-8)^{2}+4.16 .7=2^{9}, \quad K=16.16(16-8)=2^{11}, \quad L=2^{16},
$$

and hence

$$
X=-2\left(\frac{6}{7}\right)^{3}, \quad Y=\frac{2}{4} \frac{4}{9} .
$$

Next, at the point $\left(2^{8} .11^{-3}, 3.2^{5} .11^{-2}\right)$, where $D=0, \varpi=0$ cut one another, given on $\varpi=0$ by taking the parameter $\tau$ to be unity, and on $D=0$ by taking the parameter $\mu$ to be $2^{4} / 11$, we have

$$
\frac{u}{v}=-1, \quad \frac{u}{v}=\frac{1+\xi(\epsilon-1)^{\frac{1}{2}}}{1-\zeta(\epsilon-1)^{\frac{1}{2}}}=1,1, \frac{1+i \sqrt{ } 2}{1-i \sqrt{ } 2}, \frac{1-i \sqrt{ } 2}{1+i \sqrt{ } 2}
$$

thus, if $L>0$, there are two conjugate complex roots of the original quintic, and three real roots, one of these repeated; and, if $L<0$, and

$$
u=-\frac{1}{2}(w+i \sigma), \quad v=-\frac{1}{2}(w-i \sigma),
$$

there are five real roots given by $v=0, \sigma=0$ twice, and $\sigma / w= \pm \sqrt{ } 2$.

Finally, the origin corresponds to $L=0$, and a canonical form

$$
A u^{5}+5 E u v^{4}+F v^{5},
$$

where $u, v$ are real linear forms, and $A, E, F$ are real. This cannot have five real roots, since the derivative $A u^{4}+E v^{4}$ can vanish only for two real values of $u / v$, and that only when $E / A$ is negative. By examining the signs of $A u^{5}+5 E u v^{4}+F v^{5}$ for the values of $u / v$ for which the derivative vanishes, we easily prove that the condition for three real roots is

$$
A F^{4}+2^{8} E^{5} \leqslant 0 ;
$$

since

$$
J=A^{2} F^{r 2}, \quad K=-2 A^{3} E^{5},
$$

this is the same as

$$
D=J^{2}-2^{7} K \leqslant 0 ;
$$

when $D>0$ there is only one real root. In our figures, the curve we have denoted by $D=0$ passes through the origin ; but the discriminant $D$ of the quintic does not necessarily vanish when $L=0$, being in fact equal to

$$
\frac{2^{8} \xi^{4}}{\varpi^{2}}\left[\left(\eta^{2}-\xi\right)^{2}-2^{7} \xi^{2} \eta\right]
$$

and the vanishing of $\varpi$ allows $D$ to be positive or negative. In fact 
140 INVARIANTS OF A BINARY QUINTIC AND THE REALITY OF ITS ROOTS.

$L=0, K \neq 0$, gives $X=0, Y=0$ and $3 Y^{2} / 4 X=K^{2} /\left(K^{2}-J L\right)=1$. The case $L=0, K=0$ gives, on examination, $X=0, Y=$ finite.

The numbers of real roots of the quintic at the three points are then given by the diagram

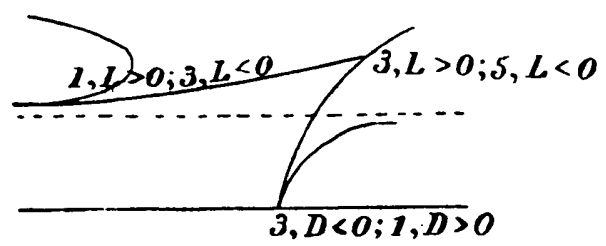

